\title{
Perspective Rectification of Integral Images Produced using Hexagonal Lens Arrays
}

\author{
E. T. Koufogiannis ${ }^{1}$ N. P. Sgouros ${ }^{1}$ M. S. Sangriotis ${ }^{1}$ \\ ${ }^{1}$ Department of Informatics and Telecommunications University of Athens, \\ Panepistimiopolis Athens, Greece 15784 \\ \{efthimis,nsg,sagri\}@di.uoa.gr
}

\begin{abstract}
Nowadays a plethora of three-dimensional (3D) systems claim users' increasing preference for delivering 3D content. In recent years an increasing number of systems utilize autostereoscopic techniques and hence provide glasses free viewing and adequate resolutions in full color. In addition certain autostereoscopic techniques like Integral Imaging provide twodimensional (2D) parallax and further increase anticipation for high fidelity 3D content viewing to professional and home users. In this paper we extend the results of previous research to provide perspective distortion rectification for Integral Images generated using hexagonal instead of square lenses. In the proposed method we demonstrate the utilization of a fundamental property of distorted coplanar hexagonal lattices in the image rectification framework that improve the rectification accuracy and robustness. Finally we provide experimental results over a number of images to evaluate the accuracy of the method using objective metrics.
\end{abstract}

\section{INTRODUCTION}

Integral Imaging (InIm) is currently considered as one of the most successful techniques for delivery of 3D multimedia content [1]. The technique extends the features of currently existing autostereoscopic techniques as it inherently provides 2D parallax to the viewer. The basic InIm acquisition setup consists of a charged coupled device (CCD) and a lens array (LA)[2], [3] which projects a real world scene on the CCD, forming a number of Elemental Images (EI) depicting different parts of the scene. Additional details of the InIm display and acquisition processes can be found in [2], [3].

In Fig. 1(a),(b) we show the basic lens types (spherical, hexagonal and square) in their basic configurations (square and hexagonal) which are used in various acquisition and display setups. From the different lens types and their configurations it can be easily derived that the use of spherical lenses creates gaps between the lenses in all possible configurations hence presenting low fill factors, while the use of square or hexagonal lenses provide high fill factors. Moreover hexagonal lenses in a hexagonal configuration reach a $100 \%$ fill factor while preserving a spherical contour [4], [5].

In this paper we address the problem of perspective distortion introduced due to small misalignments which occur when placing a hexagonal LA over the CCD in the acquisition setup. These misalignments transform the hexagonal EIs into irregular hexagons. An ideal and distorted InIm of a dice are shown in Figs. 1(e), 1(f) respectively. Practically these aberrations result in poor 3D representation through an InIm display, or a deformed 3D object after applying a 3D reconstruction procedure [6].

Related work in the field is focused on InIm capturing systems where square lenses were used using a square configuration [7], [8], [9]. The method proposed in this paper is based in the generalized rectification framework presented in [9].

The basic concept of the proposed methodology is to utilize all of the EIs boundaries in the rectification process. This approach increases the reliability and robustness of the algorithm taking under consideration the InIm structural components. In section II we outline the line segment detection and registration process while in section III we summarize the basic projective geometry principles used for rectifying the Integral Image. In section IV we present the implementation details of the proposed rectification method. In section $\mathrm{V}$ we utilize an objective geometric consistency metric to evaluate the performance of the proposed algorithm along with a number of conclusions.

\section{Line Segment Detection And Registration}

The first step of the whole framework is the detection of line segments that belong to EI boundaries. For this reason

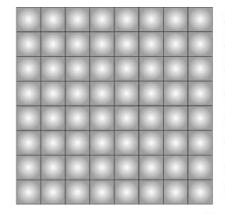

(a)

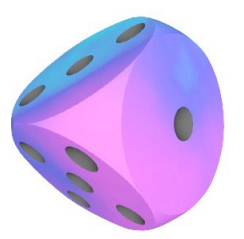

(c)
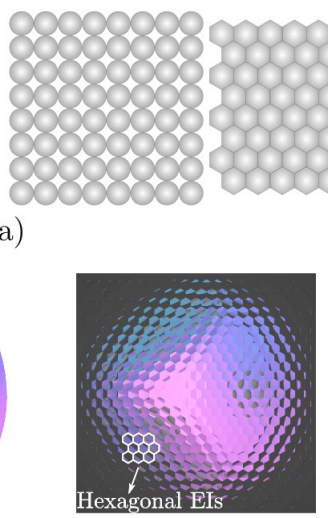

(d)

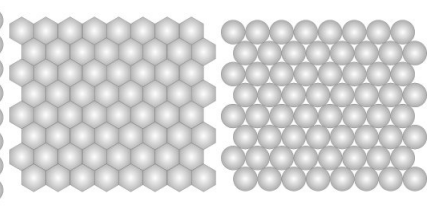

(b)

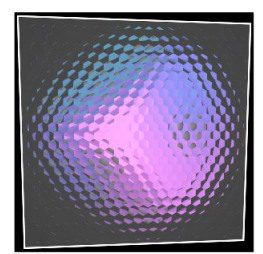

(e)
Fig. 1. (a) LAs with square packing of lenses, (b) LAs with hexagonal packing. (c) Photograph of a dice. (d) The image that would ideally be acquired. (e) The acquired InIm due to misalignments between the Lens Array and the CCD. The border in (f) is shown for illustration purposes. 


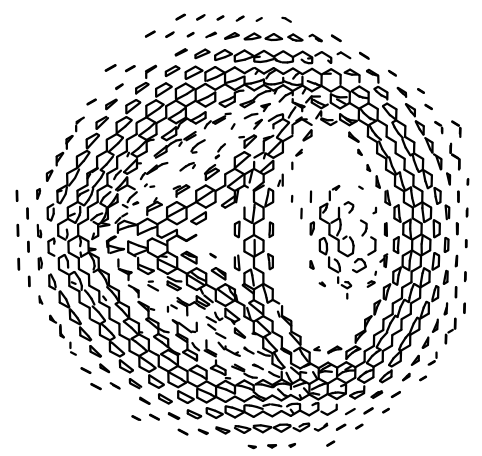

(a)

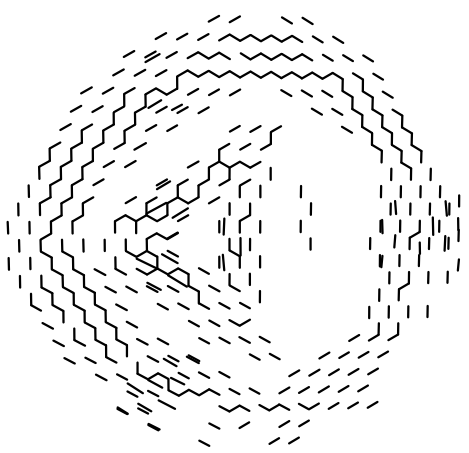

(b)

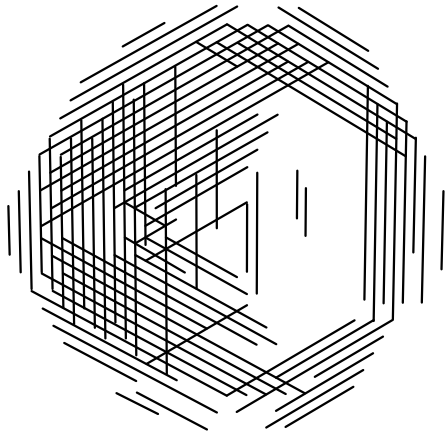

(c)

Fig. 2. (a) LSD results. (b) Detections results after removing invalid segments. (c) Fitted segments

we use the LSD algorithm[10] due to the fact that it does not require any parameter setting and it is not easily deceived by the random texture of the EI content.

The algorithm outputs a sequence of line segments $\left\{l_{i}\right\}$, each represented by its start and end points denoted respectively by coordinates $\left(x_{s_{i}}, y_{s_{i}}\right)$ and $\left(x_{e_{i}}, y_{e_{i}}\right)$.

The detected segments after applying the LSD algorithm are shown in Fig. 2(a). It can be observed in Fig. 2(a) that the sequence $\left\{l_{i}\right\}$ contains segments corresponding to the grid boundaries. It also contains segments that belong to the scene content.

Moreover as shown in Fig. 2(a) the direction angles of the grid segments form three populations separated by an angular distance of around $60^{\circ}$, while the rest of the segments have random direction angles. Since the shape of an ideal EI is a regular hexagon the directions of the three pairs of line segments forming the hexagon outline should exhibit $-30^{\circ}$, $30^{\circ}, 90^{\circ}$ angles with the $\mathrm{x}$-axis. However in the distorted InIm these values are subject to moderate deviations.

\section{A. Invalid Segment Identification and Rejection}

In order to reject most of the misdetected line segments we evaluate the direction angle sequence of $\left\{l_{i}\right\}$ and subsequently we calculate the histogram of the direction angle values in the range $\left(-45^{\circ}, 135^{\circ}\right]$ as shown in Fig. 3(a) to avoid folding around $90^{\circ}$. Each of the three lobes in the resulting histogram of Fig. 3(a) corresponds to segments forming the EI grid. These three lobes are labeled based on their peak angle values ie $-30^{\circ}, 30^{\circ}, 90^{\circ}$. The remaining histogram bins contain small populations of line segments corresponding to content within the EIs.

The populations of these three basic directions are automatically isolated using the following process:

i. The calculated histogram is partitioned using the median points between the lobes thus forming 3 single lobe histogrames.

ii. For each of the three histograms we calculate the bin having the maximum count. We repartition this histogram population based on the calculated maximum value (Fig. 3(b), 3(c), 3(d)). iii. We apply an optimum threshold algorithm (OTA) [11] on each of the repartitioned populations of step ii to calculate two thresholds $t_{l}$ and $t_{r}$. These thresholds separate these populations from the undesired line segments.

iv. Finally we disregard all segments with lengths less than $10 \%$ of the maximum segment length to minimize the uncertainty introduced by small noisy segments.

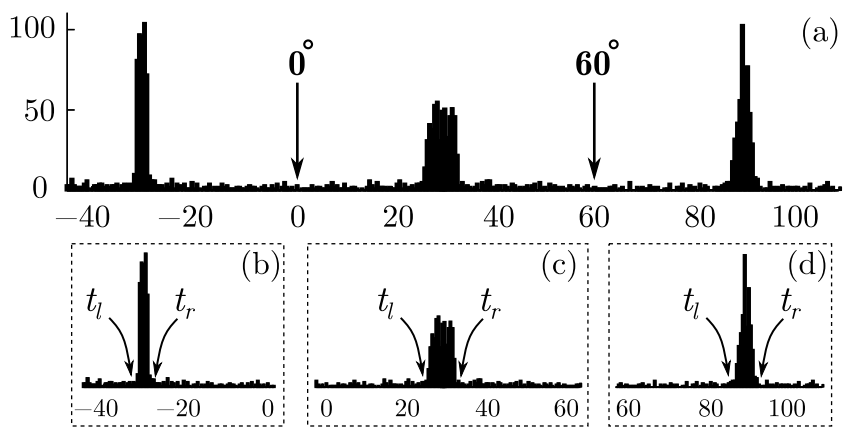

Fig. 3. (a) The histogram of all the segment angles. (b) (c) and (d) Histograms corresponding to angle ranges $\left(-45^{\circ}, 0^{\circ}\right],\left(0^{\circ}, 60^{\circ}\right]$ and $\left(60^{\circ}, 135^{\circ}\right]$.

Comparing Fig. 2(a) with Fig. 2(b) we notice that most of the invalid line segments not belonging to the EI boundaries are rejected. A small number of remaining line segments that are misregistered or belong to the EI content swill be further reduced in the next stage of the proposed method.

\section{B. Clustering of Collinear Segments}

Subsequently a hierarchical clustering procedure is used to classify the line segments of each group into collinear clusters. For this purpose we use a symmetric dissimilarity measure [9] $d_{m}\left(l_{i}, l_{j}\right)$ for every pair of segments $\left(l_{i}, l_{j}\right)$ given by (1):

$$
d_{m}\left(l_{i}, l_{j}\right)=\max \left\{d\left(s_{i}, l_{j}\right), d\left(e_{i}, l_{j}\right), d\left(s_{j}, l_{i}\right), d\left(e_{j}, l_{i}\right)\right\}
$$

where $d(p, l)$ represents the distance of a point $p$ from the line segment $l$ and $\left(s_{i}, e_{i}\right)$ and $\left(s_{j}, e_{j}\right)$ are the coordinates of the start and end points of each line segment. We also extend this 
definition of $d_{m}\left(l_{i}, l_{j}\right)$ to the dissimilarity measure between two clusters of collinear segments $C_{p}$ and $C_{q}$, as:

$$
d_{m}\left(C_{p}, C_{q}\right)=\min \left\{d_{m}\left(l, l^{\prime}\right)\right\}, l \in C_{p}, l^{\prime} \in C_{q}
$$

A version of the hierarchical clustering method, called single-linkage clustering [12], is used to populate the different clusters and the procedure finishes as soon as the minimum dissimilarity measure of the clusters exceeds an automatically calculated threshold value $d_{t h}$ [9].

After the clusters are formed, a line is fitted through the segment endpoints in each cluster. In Fig. 2(c) the fitted lines are plotted. The plotted lines are limited to the extent of the line segments in each cluster for illustration purposes. At this point three groups of line equations are derived, denoted as $L_{-30^{\circ}}, L_{30^{\circ}}, L_{90^{\circ}}$ which will be used in the InIm rectification process.

\section{IMPLEMENTATION DETAILS}

As already mentioned the ideal $I_{i d}$ and the acquired InIm $I_{a c}$ are related through a perspective transformation $H$. According to Liebowitz [13] this transformation can be decomposed to a product of three matrices so that $H=H_{p} H_{a} H_{s}$ where $H_{p}, H_{a}$ and $H_{s}$ represent a pure perspective, an affine and a similarity transformation respectively. The similarity transformation $H_{s}$ in our case is purely rotational since we are not interested in translation and scaling. Therefore the matrices are defined as:

$$
\begin{gathered}
H_{p}=\left(\begin{array}{ccc}
1 & 0 & 0 \\
0 & 1 & 0 \\
l_{1} & l_{2} & l_{3}
\end{array}\right), H_{a}=\left(\begin{array}{ccc}
\frac{1}{\beta} & -\frac{\alpha}{\beta} & 0 \\
0 & 1 & 0 \\
0 & 0 & 1
\end{array}\right) \\
H_{s}=\left(\begin{array}{ccc}
\cos \theta & \sin \theta & 0 \\
-\sin \theta & \cos \theta & 0 \\
0 & 0 & 1
\end{array}\right)
\end{gathered}
$$

where $l_{1}, l_{2}, l_{3}$ are the vanishing line's [14] parameters, $\alpha, \beta$ are the affine correction parameters.

At this point we should also mention a basic feature of projective geometry [14] where each perspectively distorted plane has one corresponding vanishing line. An important resulting property is that the vanishing points corresponding to groups of parallel lines on a plane are collinear. Therefore the three sets of parallel lines corresponding to a hexagon will meet the plane's vanishing line in 3 collinear vanishing points $V_{1}, V_{2}$ and $V_{3}$ as shown in Fig. 4(b).

Due to segment detection errors that occur in a real world scenario the grid lines in $I_{a c}$ converge within a small area instead of a single point. To compensate we calculate the median of all intersection points as the vanishing point of each line set. Median values ensure that outliers have no effect over the calculation of the vanishing point coordinates. A subsequent least squares line fitting through the three estimated vanishing points is performed to calculate the vanishing line.

The resulting transformation matrix $H_{p}$ is applied on the three line equation sets $L_{-30^{\circ}}, L_{30^{\circ}}, L_{90^{\circ}}$ producing three new sets where each one has its lines approximately parallel since

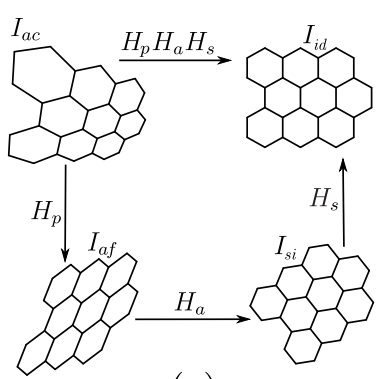

(a)

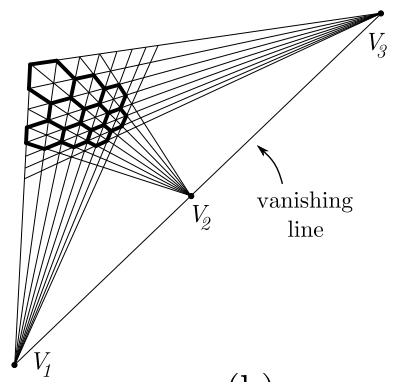

(b)

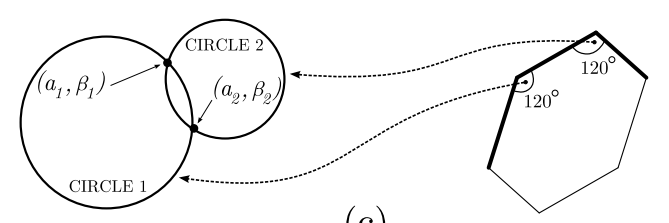

(c)

Fig. 4. The sequential rectification transformations of a hexagonal grid. The three vanishing points of the distorted hexagonal grid.

multiplication with matrix $H_{p}$ removes the pure projective distortion component $H_{p}$. In this stage, line parallelism has been restored, but length and angle ratios are inconsistent with the length and angle ratios in the undistorted InIm.

To calculate the metric correction parameters $\alpha, \beta$ we construct two circle equations exploiting geometric properties of the distorted objects in the acquired image [13]. It is basic knowledge that the internal angles of a regular hexagon are $120^{\circ}$ each. Therefore the known angles constraint [13] is applied twice on the affine image $I_{a f}$ : between the average line directions corresponding to $90^{\circ}$ and $30^{\circ}$ and between the average line directions corresponding to $30^{\circ}$ and $-30^{\circ}$ as shown in Fig. 4(c). Each application generates the equation of a circle on the $\alpha \beta$ plane. From the two circle intersection points $\left(\alpha_{1}, \beta_{1}\right),\left(\alpha_{2}, \beta_{2}\right)$ we choose $\left(\alpha_{i}, \beta_{i}\right)$ so that $\beta_{i}>0$ [13].

For the estimation of $H_{s}$ which defines a similarity transformation between $I_{s i}$ and $I_{i d}$ we calculate the rotation angle $\theta$ in such a way that the grid lines of $I_{s i}$ become parallel to the grid lines in $I_{i d}$.

\section{EXPERIMENTS, RESUlTS AND CONCLUSIONS}

\section{A. Experimental Parameters}

Using the methodology described in [15] we generated a number of different InIms with hexagonal EIs. We distorted these images with known matrices $H_{p}, H_{a}, H_{s}$ and introduced three different noise levels. We follow this approach because it offers full control over the displayed scene complexity and noise characteristics. It also gives us additional a-priori knowledge of the ideal values of parameters $l_{1}, l_{2}, l_{3}, \alpha, \beta, \theta$.

In order to measure the geometric consistency of the proposed method we consider the three line sets $L_{A}, L_{B}, L_{C}$ corresponding to $90^{\circ}, 30^{\circ},-30^{\circ}$ respectively and introduce two parameters that can be algorithmically measured on the rectified grid: 
i. The obtuse angles formed between all the $L_{A}$ lines and all the $L_{B}$ lines, as well as the obtuse angles formed between all the $L_{B}$ lines and all the $L_{C}$ lines. We form the sequence $\{\omega\}$ of all the collected angle values which should be $120^{\circ}$ in the ideal case since they correspond to internal hexagon angles.

ii. We measure the length of the segments formed on each line of the $L_{A}$ set when intersected by all of the lines in $L_{B}$. In the same way we find the segments formed on each line of the $L_{A}$ set when intersected by all of the lines in $L_{C}$. The process is repeated for the segments formed on each $L_{B}$ and $L_{C}$ line. Ideally these segments should have the same length. The sequence of all these length values is constructed and is subsequently normalized by dividing all the elements with the average segment length. The resulting sequence $\{s\}$ contains the normalized segment lengths which ideally should be equal to 1 .

\section{B. Results}

In this section we present the results of the measurements performed for a characteristic InIm of the test set, which is depicted in Fig. 1(f). The statistical evaluation results for both sequences $\{\omega\}$ and $\{s\}$ and different noise levels are summarized in Table I. We can observe that the angle values span a very narrow region around the nominal value of $120^{\circ}$ with a significantly small standard deviation. The same observation holds for the normalized segment lengths, where the mean length is 1 and the standard deviation 0.01. It should be noted that the standard deviation of the normalized segment lengths contains information regarding the introduced error, since after the normalization process the mean length value will become 1 .

TABLE I

GEOMETRIC CONSISTENCY EVALUATION

\begin{tabular}{lcc}
\hline & $\bar{\omega}_{i} \pm \sigma_{\omega_{i}}$ & $\sigma_{\lambda_{i}} \%$ \\
\hline noiseless & $119.97^{\circ} \pm 0.05^{\circ}$ & $1.0 \%$ \\
\hline $30 \mathrm{~dB}$ & $119.96^{\circ} \pm 0.07^{\circ}$ & $1.1 \%$ \\
\hline $25 \mathrm{~dB}$ & $120.0^{\circ} \pm 0.1^{\circ}$ & $1.5 \%$ \\
\hline $20 \mathrm{~dB}$ & $119.9^{\circ} \pm 0.2^{\circ}$ & $2.5 \%$ \\
\hline
\end{tabular}

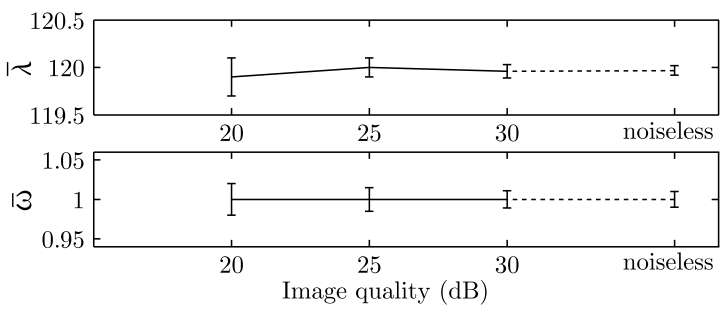

Fig. 5. Parameters $\{\omega\}$ and $\{s\}$ and their respective errors for different noise levels

\section{Conclusions}

The results show that only small deviations from the ground truth geometric consistency parameters occur. The proposed statistical approach ensures that a significant number of outliers is rejected. Furthermore the use of the line segment clustering and fitting scheme ensures that possible remaining outliers do not influence the correct reconstruction of the line sets. The proposed method constitutes a valuable and robust tool for the rectification of InIms where hexagonal LAs are used.

\section{ACKNOWLEDGMENT}

This research has been co-financed by the European Union (European Social Fund) and Greek national funds through the Operational Program "Education and Lifelong Learning" of the National Strategic Reference Framework (NSRF) Research Funding Program: Heracleitus II. "Investing in knowledge society through the European Social Fund."

\section{REFERENCES}

[1] J.-Y. Son and B. Javidi, "Three-dimensional imaging methods based on multiview images," J. Display Technol., vol. 1, no. 1, p. 125, Sep 2005.

[2] J.-H. Park, Y. Kim, J. Kim, S.-W. Min, and B. Lee, "Three-dimensional display scheme based on integral imaging with three-dimensional information processing," Opt. Express, vol. 12, no. 24, pp. 6020-6032, Nov 2004.

[3] J.-S. Jang and B. Javidi, "Formation of orthoscopic three-dimensional real images in direct pickup one-step integral imaging," Optical Engineering, vol. 42, no. 7, pp. 1869-1870, 2003.

[4] D. Kuang, X. Zhang, M. Gui, and Z. Fang, "Hexagonal microlens array fabricated by direct laser writing and inductively coupled plasma etching on organic light emitting devices to enhance the outcoupling efficiency," Appl. Opt., vol. 48, no. 5, pp. 974-978, Feb 2009.

[5] M.-C. Chou, C. Pan, S. Shen, M.-F. Chen, K. Lin, and S.-T. Wu, "A novel method to fabricate gapless hexagonal micro-lens array," Sensors and Actuators A: Physical, vol. 118, no. 2, pp. 298 - 306, 2005.

[6] G. Passalis, N. Sgouros, S. Athineos, and T. Theoharis, "Enhanced reconstruction of three-dimensional shape and texture from integral photography images," Appl. Opt., vol. 46, no. 22, pp. 5311-5320, Aug 2007.

[7] N. P. Sgouros, S. S. Athineos, M. S. Sangriotis, P. G. Papageorgas, and N. G. Theofanous, "Accurate lattice extraction in integral images," Opt. Express, vol. 14, no. 22, pp. 10 403-10409, Oct 2006.

[8] K. Hong, J. Hong, J.-H. Jung, J.-H. Park, and B. Lee, "Rectification of elemental image set and extraction of lens lattice by projective image transformation in integral imaging," Opt. Express, vol. 18, no. 11, pp. 12002-12 016, May 2010.

[9] E. T. Koufogiannis, N. P. Sgouros, and M. S. Sangriotis, "Robust integral image rectification framework using perspective transformation supported by statistical line segment clustering," Appl. Opt., vol. 50, no. 34, pp. H265-H277, Dec 2011.

[10] R. von Gioi, J. Jakubowicz, J.-M. Morel, and G. Randall, "Lsd: A fast line segment detector with a false detection control," Pattern Analysis and Machine Intelligence, IEEE Transactions on, vol. 32, no. 4, pp. 722-732, april 2010.

[11] M. Unser and M. Eden, "Multiresolution feature extraction and selection for texture segmentation," Pattern Analysis and Machine Intelligence, IEEE Transactions on, vol. 11, no. 7, pp. 717 -728, jul 1989.

[12] S. Theodoridis and K. Koutroumbas, Pattern Recognition, Third Edition, 3rd ed. Academic Press, 2006.

[13] D. Liebowitz and A. Zisserman, "Metric rectification for perspective images of planes," in Proceedings. 1998 IEEE Computer Society Conference on Computer Vision and Pattern Recognition (Cat. No.98CB36231). IEEE Comput. Soc, 1998, pp. 482-488.

[14] R. I. Hartley and A. Zisserman, Multiple View Geometry in Computer Vision, 2nd ed. Cambridge University Press, 2004.

[15] S. S. Athineos, N. P. Sgouros, P. G. Papageorgas, D. E. Maroulis, M. S Sangriotis, and N. G. Theofanous, "Photorealistic integral photography using a ray-traced model of capturing optics," Journal of Electronic Imaging, vol. 15, no. 4, p. 043007, 2006. 